BULL. AUSTRAL. MATH. SOC.

VOL. 36 (1987) $345-347$

\title{
THE GROWTH OF STRATFIED GROUPS
}

\section{NichOLAS BILLINGTON}

In this thesis we deal with stratfied groups. These are pairs $(G, S)$ where $G$ is a group and $S=\left(S_{n}\right)^{\prime} \geq 0$ is an increasing sequence of finite subsets of $G$ whose union is $G$ and such that $S_{0}=\{1\}$ and $S_{m} \cdot S_{n} \subseteq S_{m+n}$ for all $m, n \geq 0$. This includes finitely generated groups $G$ where $S_{n}$ is the set of elements in $G$ whose lengths are at most $n$ with respect to a specified finite generating set of $G$. The growth of the stratfied group $(G, S)$ is the power series, with integer coefficients

$$
(G, S)(z)=1+\sum_{n \geq 1}\left|S_{n}-S_{n-1}\right| z^{n}
$$

If $S_{m} \cdot S_{n}=S_{m+n}$ for all $m, n \geq 0$ then $S_{1}-S_{0}$ is a finite generating set for $G$ and $(G, S)(z)$ is the growth of $G$ with respect to the finite generating set $s_{1}-S_{0}$.

Received 12 December, 1986. Thesis submitted to La Trobe University, May, 1986. Degree approved October 1986. Supervisor: Dr. G. Davis.

Copyright Clearance Centre, Inc. Serial-fee code: 0004-9727/87 $\$ A 2.00+0.00$. 
The first chapter of the thesis deals with the relationship between stratfied groups and graded algebras over a field. In particular it is shown that associated with every stratfied group $(G, S)$ there is a graded algebra whose Hilbert-Poincare series is $(G, S)(z)$.

The rationality of growth series is considered in chapter two where, along with other results, it is shown that certain stratifications of abelian groups yield non-rational growth series : in fact these growth series are not even algebraic over $\mathbb{Z}[z]$.

The third chapter develops formulae for the growth of free products of groups amalgamated over finite subgroups. The essential ingredient of chapter three is a formula which gives the growth of a free product of two groups in terms of the growths of its constituent factors. This latter result is established directly. In addition, an outline is provided of the way in which this result may be deduced from the corresponding result for the Hilbert-Poincare series of a coproduct of graded algebras.

The fourth chapter deals with the relationship between the growth of a group and the growth of a central subgroup of finite index. A formula for the growth of a free product amalgamated over a central subgroup of finite index in each factor is then established via the free product growth formula obtained in chapter three. This leads to explicit calculations of the growth series of the torus knot groups.

In chapter five the concept of growth of the cells of a $\mathrm{CW}$-complex is introduced. Under suitable hypotheses it is shown that the alternating sum of the growths of cells in each dimension is equal identically to 1 . In the case that a group acts in a sufficiently nice way on the $C W$-complex, the growth of the group is exactly the growth of the 0 -dimensional cells. When the relationships between the growths of cells in adjacent dimensions are known, it is then possible to write down a rational representation of the growth of the 0 -dimensional cells, and hence the growth of the associated group. 
The growth of stratfied groups

3 Redesdale Road,

Ivanhoe,

Victoria, 3079

Australia. 